Dieses Dokument ist eine Zweitveröffentlichung (Verlagsversion) / This is a self-archiving document (published version):

Adrian Ehrenhofer, Alice Mieting, Sascha Pfeil, Johannes Mersch, Chokri Cherif, Gerald Gerlach, Thomas Wallmersperger

An automatically rainproofing bike helmet through light-sensitive hydrogel meshes

Erstveröffentlichung in / First published in:

SPIE Smart Structures + Nondestructive Evaluation. Online, 27.04. - 09.05.2020 [Zugriff am 07.06.2020]. SPIE Digital Library. ISBN 978-1-51063-528-9.

DOI: https://doi.org/10.1117/12.2557728

Diese Version ist verfügbar / This version is available on:

https://nbn-resolving.org/urn:nbn:de:bsz:14-qucosa2-742187 


\section{An automatically rainproofing bike helmet through light-sensitive hydrogel meshes: design, modeling and experiments}

Ehrenhofer, Adrian, Mieting, Alice, Pfeil, Sascha, Mersch, Johannes, Cherif, Chokri, et al.

Adrian Ehrenhofer, Alice Mieting, Sascha Pfeil, Johannes Mersch, Chokri Cherif, Gerald Gerlach, Thomas Wallmersperger, "An automatically rainproofing bike helmet through light-sensitive hydrogel meshes: design, modeling and experiments," Proc. SPIE 11375, Electroactive Polymer Actuators and Devices (EAPAD) XXII, 113750N (26 May 2020); doi: $10.1117 / 12.2557728$

Event: SPIE Smart Structures + Nondestructive Evaluation, 2020, Online Only, California, United States 


\title{
An automatically rainproofing bike helmet through light-sensitive hydrogel meshes: Design, modeling and experiments
}

\author{
Adrian Ehrenhofer ${ }^{\mathrm{a}}$, Alice Mieting ${ }^{\mathrm{b}}$, Sascha Pfeil ${ }^{\mathrm{b}}$, Johannes Mersch ${ }^{\mathrm{c}}$, Chokri Cherif ${ }^{\mathrm{c}}$, Gerald \\ Gerlach $^{\mathrm{b}}$, and Thomas Wallmersperger ${ }^{\mathrm{a}}$ \\ anstitute of Solid Mechanics, Technische Universität Dresden, 01062 Dresden, Germany \\ ${ }^{\mathrm{b}}$ Institute of Solid State Electronics, Technische Universität Dresden, 01062 Dresden, Germany \\ ${ }^{c}$ Institute of Textile Machinery and High Performance Material Technology, Technische \\ Universität Dresden, 01062 Dresden, Germany
}

\begin{abstract}
For everyday cycling, one needs to carry rainproof clothing just for the case of unexpected downpours. In the present research, we present a concept for a helmet which is automatically rainproof when the rain starts. When the sun comes out, the helmet is breathable again even before it completely dries up. This functionality is provided by active hydrogel meshes. Hydrogel meshes offer great advantages due to their ability to change the aperture size with swelling and deswelling. In our current work, we present the design and modeling steps for hydrogel-layered active meshes which use (i) swelling and deswelling in hydrated state and (ii) swelling starting from the dry state. The main goal is to close the air openings of a bicycle helmet when rain starts as an automatic rainproofing. This can be achieved through the swelling of the hydrogel pNiPAAM-co-chlorophyllin in the meshes, which leads to closing when hydrated. At the same time, the light-sensitive behavior leads to opening of the apertures under direct sun exposure, i.e. when the sun appears again after the rain. We present the steps of modeling and design using the Normalized Extended Temperature-Expansion-Model (NETEM) to perform simulations in Abaqus. The model is capable of describing both the swelling of the hydrogel under light stimulus and the volume change due to hydration. It is based on the analogy between free swelling and thermal expansion and defined for nonlinear displacements. We also discuss the fabrication process of hydrogel-layered fibers and challenges in their application and simulation. As a proof of concept for hydrogel-layered meshes, we show preliminary experimental results of a poly(acrylamide)/poly(2-acrylamido-2-methyl-1-propanesulfonic acid) (PAAm/PAMPS) hydrogel with semi-interpenetrated network (SIPN) structure and its swelling capacities on a mesh. Starting from the active hydrogel meshes as presented in the current work, the next step can be smart textiles that harness the power of hydrogels: the adaptation to combinations of stimuli - like humidity, temperature and brightness - that define environments.
\end{abstract}

Keywords: Smart Bike Helmets, Hydrogels, Modeling and Simulation, Hydrogel Meshes, Finite-ElementMethod, Experiments

\section{INTRODUCTION}

Smart hydrogels with reversible swelling capacities can be combined with passive materials to form systems with unique properties. ${ }^{1}$ So far, hydrogels are mostly used in the context of artificial muscles, ${ }^{2-5}$ for sensoric purposes ${ }^{6}$ or in microfluidics. ${ }^{7-12}$ Our goal is to broaden this spectrum by using the active properties of hydrogels in different ways: Since hydrogels can be very soft and brittle, the combination with other materials that provide better mechanical functionality, is advantageous. We therefore give a deeper insight in the combined behavior of softhard active-passive composites ${ }^{13,14}$ especially the great potential of using the swelling properties perpendicular

Further author information:

Adrian Ehrenhofer: E-mail: Adrian.Ehrenhofer@tu-dresden.de

Thomas Wallmersperger: E-mail: Thomas.Wallmersperger@tu-dresden.de

Electroactive Polymer Actuators and Devices (EAPAD) XXII, edited by Yoseph Bar-Cohen,

lain A. Anderson, Herbert R. Shea, Proc. of SPIE Vol. 11375, 113750N · C 2020

SPIE · CCC code: $0277-786 \mathrm{X} / 20 / \$ 21 \cdot$ doi: $10.1117 / 12.2557728$

Proc. of SPIE Vol. $11375113750 \mathrm{~N}-1$ 
to the load direction. ${ }^{15}$ Another focus is on the hydrogels' capabilities of adapting to combinations of multiple stimuli. ${ }^{16}$

In the current work, we focus on the application of hydrogels in combination with fiber meshes. The ensuing active composites act as automatically reacting barriers. The specific problem that is addressed with these meshes can be described as follows: the design of hydrogel-coated meshes that can be applied to bicycle helmets to allow an adaptive rainproofing, see Figure 1. In the dry state, the vents are open and air can circulate through the helmet. When the structure becomes wet due to rainwater, the vents automatically close due to the hydrogel swelling.

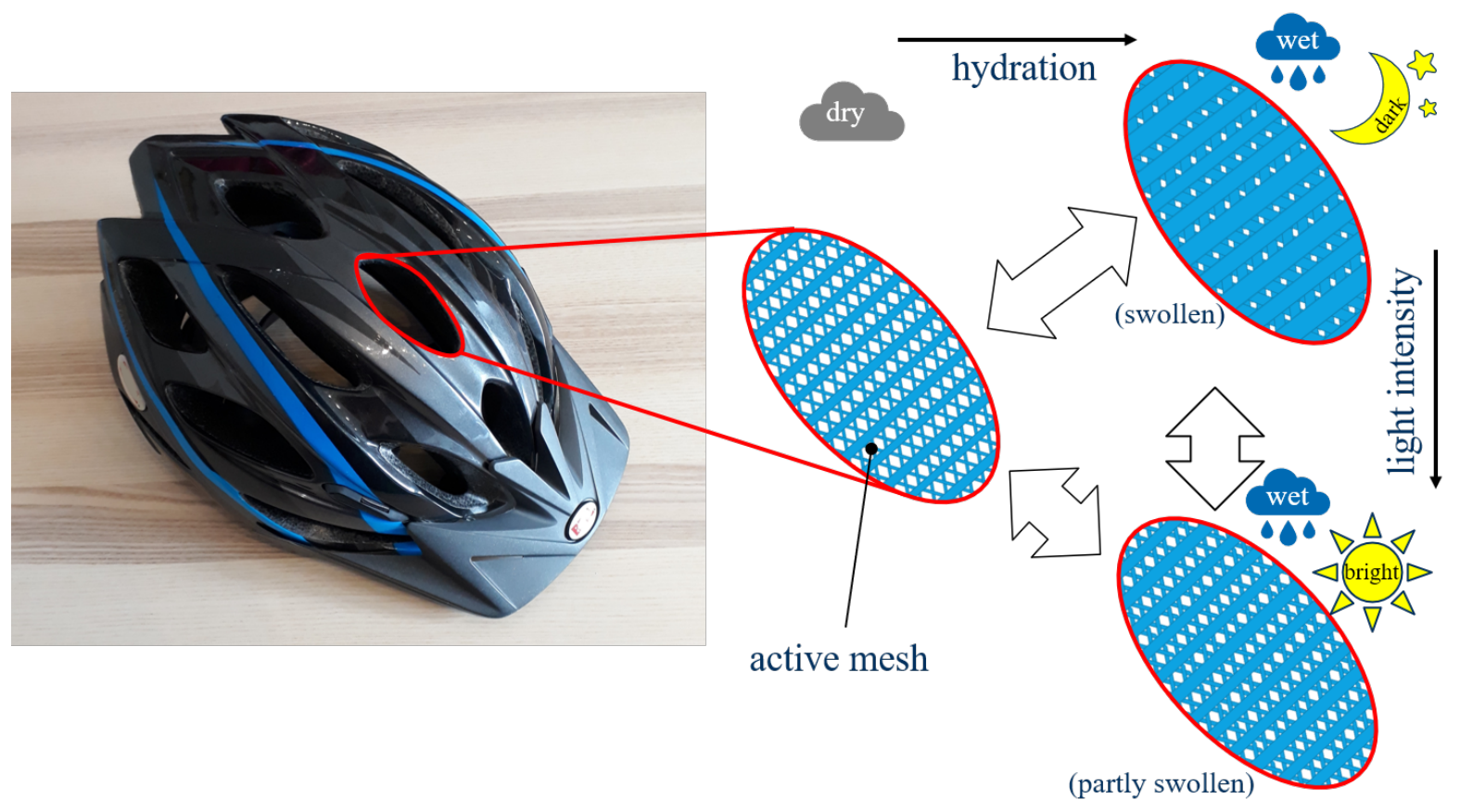

Figure 1: Helmet with openings for air circulation. In the current design concept, we present an active mesh that is fitted into these air vents. The mesh adapts to rain and sunshine to make the helm breathable or rainproof, according to the situation.

The swelling of hydrogels occurs due to different mechanisms. ${ }^{1,17}$ Depending on the number of stimuli like e.g. temperature, chemical concentrations, $\mathrm{pH}$-value or light intensity, the material is called mono- or multisensitive. ${ }^{16}$ In the current work, we focus on two specific materials: (i) The target hydrogel is a lightsensitive pNiPAAm-co-chlorophyllin copolymer presented by SuzuKI et al. ${ }^{18}$ that can be applied in the way as shown in Figure 1. Other possible candidates for this material are e.g. presented by SumARU et al., ${ }^{19}$ MAMADA et al. ${ }^{20}$ or WANG et al. ${ }^{21}$ (ii) In the current work, we use an intermediate material system of a poly(acrylamide)/poly(2-acrylamido-2-methyl-1-propanesulfonic acid) (PAAm/PAMPS) hydrogel with semiinterpenetrated network (SIPN) structure without light-sensitivity. It is applied to show the proof of concept for the adhesion of hydrogels to textiles and the added functionality of opening and closing.

Common active materials used inside or in combination with textiles are shape memory alloys, ${ }^{22}$ twisted coiled polymeric actuators ${ }^{23}$ or dielectric elastomer actuators. ${ }^{24}$ However, these actuator types require high voltage or high current power sources, which limits their use in low-cost, light-weight and portable applications. Additionally, a control unit and textile sensors for humidity, temperature or light ${ }^{25}$ have to be integrated, adding complexity, weight and cost. In contrast, hydrogel fibers or hydrogel coated textiles possess both, sensory and active properties. Hydrogel-textile composites have been proposed mostly for medical applications like tissue engineering, super absorbing textiles or drug-release patches. ${ }^{26}$ 
The modeling and simulation of hydrogel swelling allows us to perform preliminary studies without extensive need for experiments. This is especially useful for active composite mesh systems as described in this work, since (i) the production of the samples is very tedious and (ii) the parameter space including fiber thickness, mesh width, mesh type, active layer thickness etc. is large. The modeling approach that we provide in the current work allows a first design for the active mesh and can be generalized to other mesh geometries. It is based on the analogy between hydrogel swelling and thermal expansion and can be applied for arbitrary stimuli. ${ }^{27,28}$ Other approaches towards hydrogel swelling are based e.g. on the multi-field description ${ }^{29-31}$ or on the Theory of Porous Media. ${ }^{32-35}$

In the current work, we approach the behavior of active meshes from the description of a single fiber and the specific mesh setup in section 2. In this section, the preparation of a prototype coated mesh is presented as well. In the following section 3 , we show the modeling and simulation of the system based on a single fiber. Conclusions are finally drawn in section 4 .

\section{ACTIVE HYDROGEL FIBERS AND MESHES}

In order to approach the complex system of an active coated mesh, we start with the hydrogel system in section 2.1. This is followed by the idealized representation of a single fiber in section 2.2. Hydrogel and fibers build the backbone of the active mesh, which is discussed in section 2.3.

Please note, that in the current work, we focus on the elementary component of the mesh, see Figure 2: a piece of yarn that is composed of multiple filaments. For the purpose of this work, we use the term fiber for this structure.

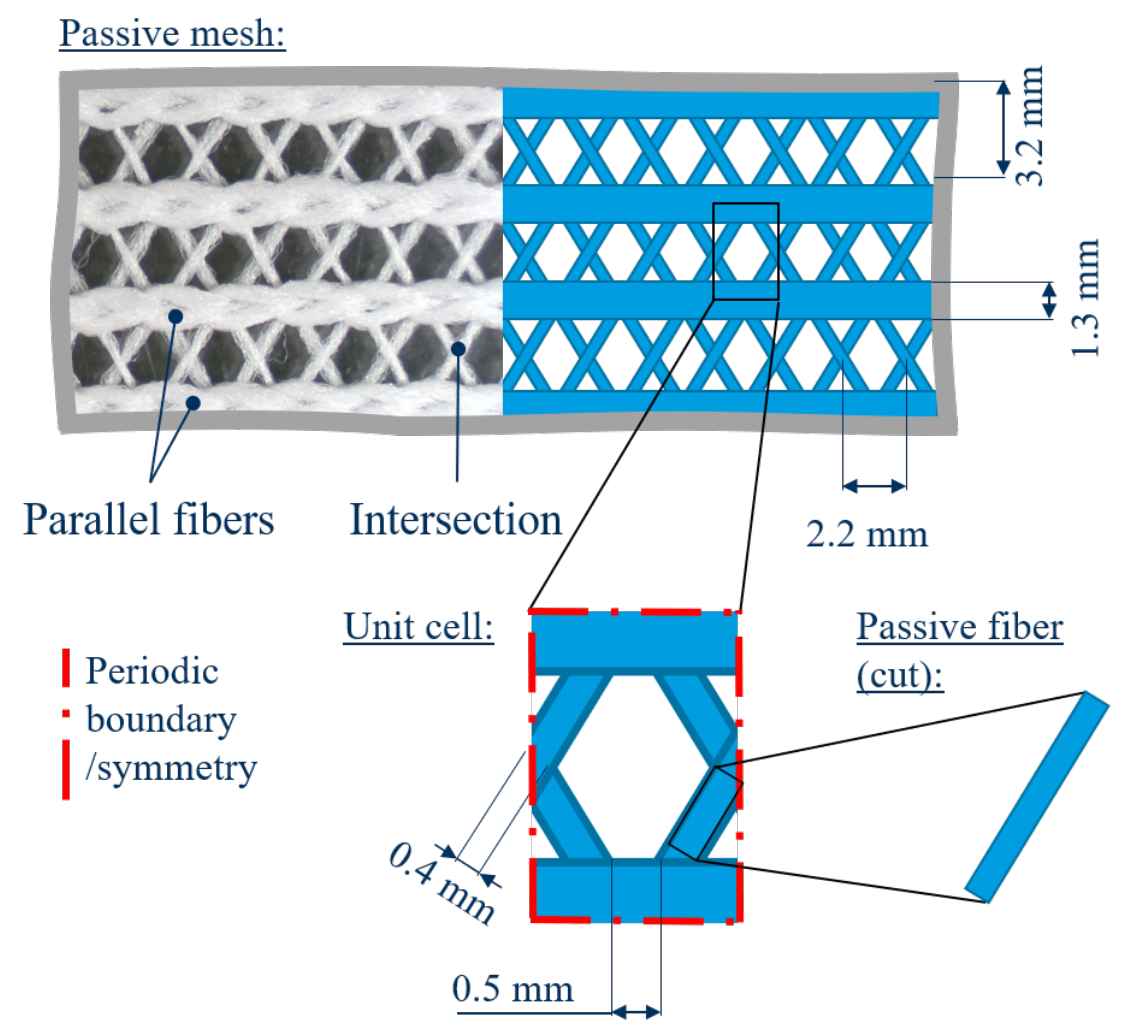

Figure 2: Two-dimensional simplification of the backbone mesh setup. The passive fiber (i.e. a piece of yarn that is composed of filaments) is the focus of the current work. Future studies will focus on the unit cell and the complete mesh. The mesh details can be found in section 2.3. The width values are mean values that were measured with Image $J{ }^{36}$ calibrated on a $1 \mathrm{~cm}$ scale. 


\subsection{Hydrogel base system for coating}

The base system that is used in the current work is an interpenetrating network of polymers called netpoly(acrylamide)-sipn-poly(2-acrylamido-2-methyl-1-propanesulfonic acid). The material combines the properties of (i) a mechanically stable poly(acrylamide) hydrogel ${ }^{37}$ with (ii) poly(2-acrylamido-2-methyl-1-propanesulfonic acid) which offers fast swelling with a high swelling ratio due to its anionic polymerchains. ${ }^{16,38,39}$

For the synthesis, acrylamide (22.61 mg, Sigma Aldrich) and N,N'-methylene-bis-acrylamide (0.21 mg, Sigma Aldrich) were dissolved in double deionized water $\left(\mathrm{ddH}_{2} \mathrm{O}, 82 \mu \mathrm{L}\right)$ and $15 \mathrm{wt} \%$ poly (2-acrylamido-2-methyl-1propanesulfonic acid) (200 $\mathrm{L}$, Sigma Aldrich) were added. Free radical polymerization was started by adding ammonium peroxodisulfate $(0.20 \mathrm{mg}$, Sigma Aldrich) and N,N,N',N'- tetramethylethylenediamine $(1 \mu \mathrm{L}$, Carl Roth). The pre-gel was applied to the mesh with a pipette and polymerized overnight at $40^{\circ} \mathrm{C}$.

For a rough estimate of the swelling capabilities, the mass swelling degree $Q$ of a material sample was determined. It showed a mass swelling degree of $Q=\left(m-m_{0}\right) / m_{0} \approx 79$, where $m_{0}=0.2 \mathrm{mg}$ is the dry mass acquired after drying at room temperature overnight and $m=16 \mathrm{mg}$ is the mass of the swollen sample. This translates into an isotropic swelling strain of $\varepsilon^{\mathrm{H}} \approx 4.3$.

\subsection{Single fiber setup}

A hydrogel-coated fiber consists of a passive fiber material (i.e. a yarn that is composed of multiple filaments) and its active coating (hydrogel), see Figure 3a. However, the specific nature of connection between polymeric coating and polymeric fiber depends on the material pairing. It can be distinguished between two extreme cases according to the hydrophilic properties of the fibers:

In Case I (separated), the hydrogel only forms outside of the fiber. It then acts as a thin coating layer similar to an isolation layer of a cable - with additional swelling capacities.

In Case II (interpenetrating) the hydrogel forms both inside and outside of the fiber. This can be the case when the fibers allow the inclusion of water - and therefore also of pre-polymer/pre-gel solution - similar to a porous medium. When the polymerization/gelation is started, the hydrogel forms inside and outside of the fiber. These parts of the network - inside the fiber and outside in the coating - are connected. This might lead to a much better adhesion of the coating compared to the aforementioned case.

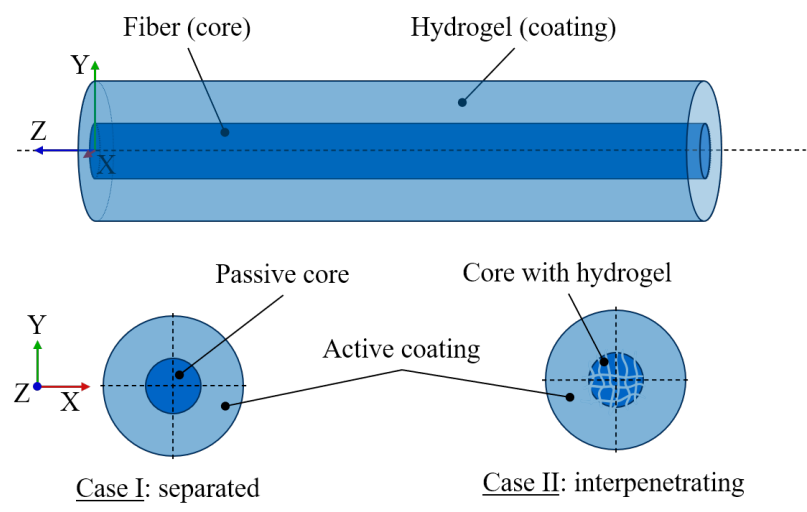

(a) Single fiber in Figure 2.

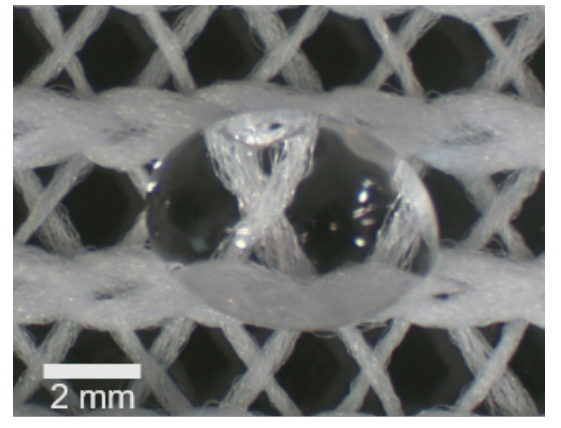

(b) Drop on a mesh unit.

Figure 3: (a) Simplified version of a single fiber for Case I and Case II. (b) Fiber in the context of the mesh with a water drop.

Between those extreme cases, there can also be intermediate cases where parts of the polymer chains of the coating range into the fibers. In the current material combination, a drop of water on the mesh is slowly soaked into the fiber. We can therefore assume that the material forms similar to Case II. 


\subsection{Preparation of the active mesh and swelling experiments}

The experimental base system that is regarded in the current work is presented in Figure 2. The textile is a warp knit manufactured on a Laboratory Raschel Machine Racop TR6 (LIBA Maschinenfabrik GmbH, Germany) with a gauge of $12 \mathrm{E}$, a single needle bed and three active guide bars. The stitch construction is a jersey stitch with an additional chain stitch made from polyethylene yarn (yarn count: 20 tex). After stretching the stitch course density and the stitch wale density are $4.5 \mathrm{~cm}^{-1}$ and $3.1 \mathrm{~cm}^{-1}$, respectively. The mesh is put into a stretching frame to simulate the integration to the helmet vents, see Figure 4.
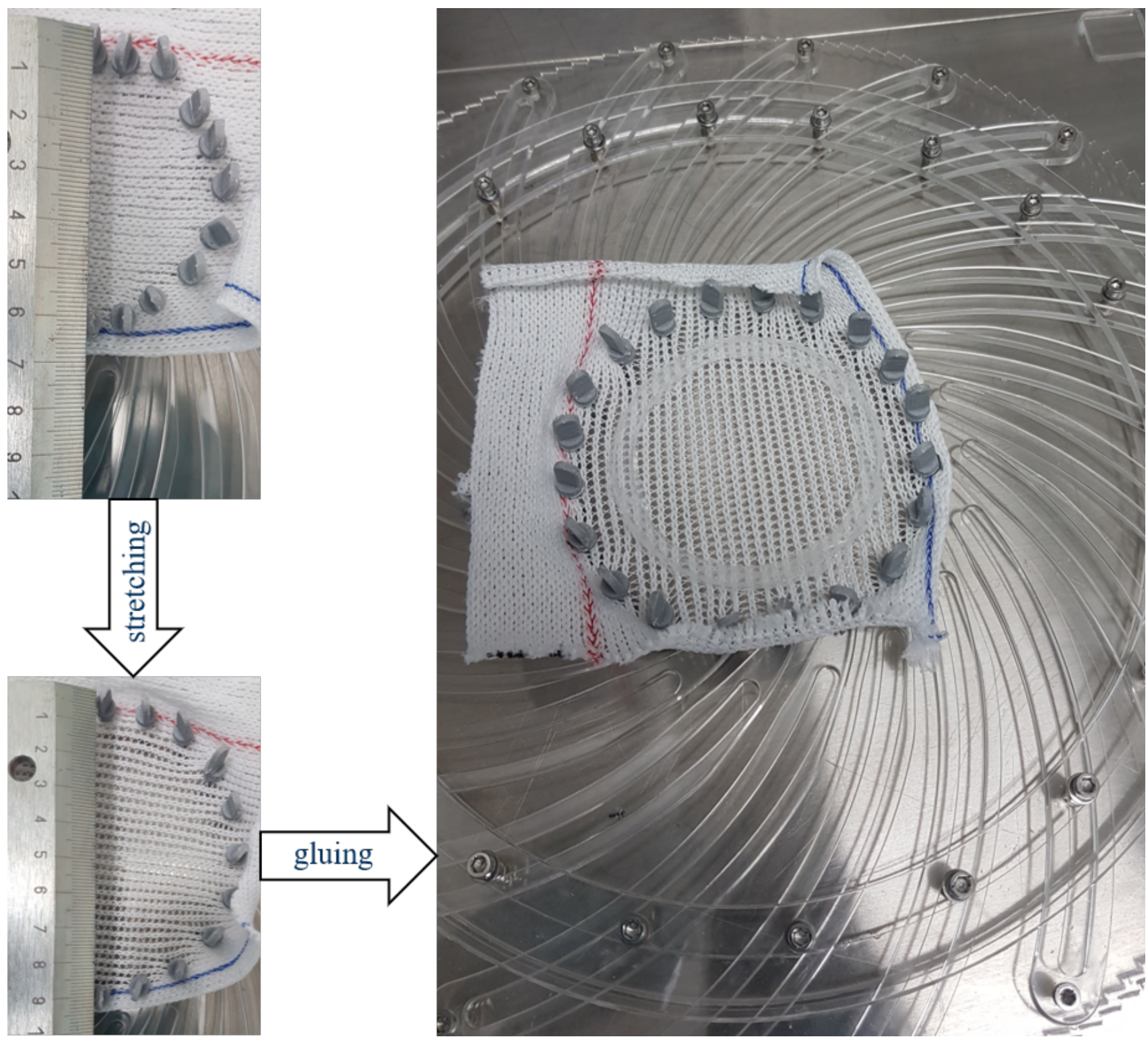

Figure 4: The initial mesh is stretched and glued onto a polymer ring. The mesh inside the ring serves as the backbone for the coating of the hydrogel, which finally leads to the active mesh. 
In order to conduct swelling experiments, the hydrogel described in section 2.1 is applied to the mesh. After the polymerization, swelling experiments are performed. Figure 5a shows the subsequent swelling of the hydrogel mesh in a water bath. The large volume increase in the respective part of the mesh finally causes a closing of the mesh pores, as it is the goal of the current work.

In Figure 5b, a drop of water hits the mesh. The fast swelling results in the closing of the pores, so that consecutive drops cannot pass. The preliminary results for this hydrogel-fiber combination also support the thesis that the combination is according to Case II from Figure 3a, i.e. the hydrogel forms both inside and outside of the fiber.

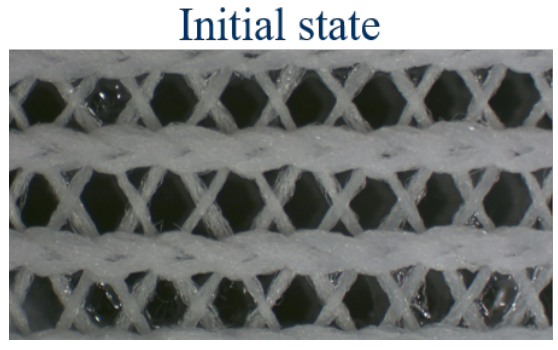

Initial state

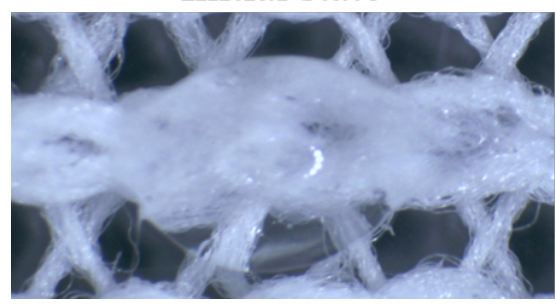

After 2 minutes

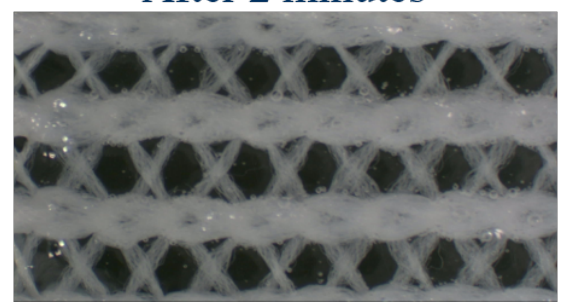

(a) Sample in a water bath.

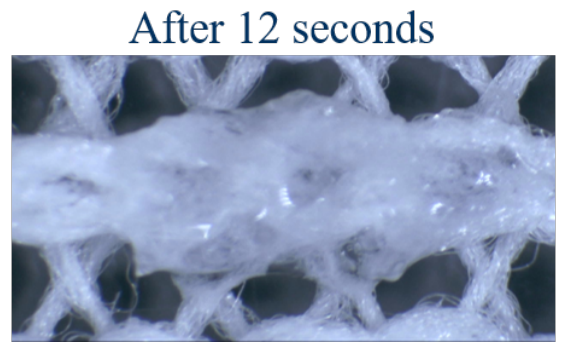

(b) Sample with drop of water.

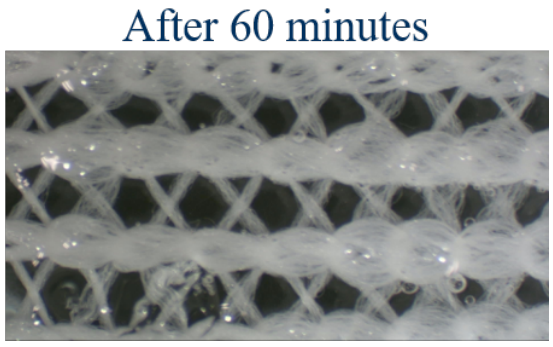

After 24 seconds

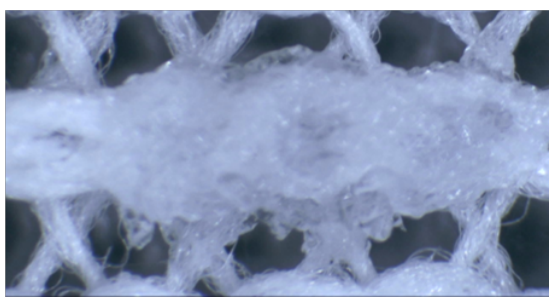

Figure 5: Microscopy images of the hydrogel coated mesh. The initially dried mesh sample is (a) immersed in a water bath and the swollen meshes after 2 and 60 minutes are presented and (b) a drop of water is added and the fast swelling response of the hydrogel after 12 and 24 seconds is presented. 


\section{MODELING AND SIMULATION WITH THE TEMPERATURE EXPANSION MODEL}

In order to design an active mesh system, modeling considerations have to be performed, see section 3.1. The current model for active swelling behavior of hydrogels is then applied in the commercial Finite-Element tool Abaqus to perform numerical simulations, see section 3.2. Here, the focus lies on the difference between the aforementioned Case I and Case II, i.e. the properties of a single fiber with (i) a thin coating and (ii) a hydrogel spanning both fiber and coating.

In the current work, we focus on three states for the hydrogel, see Figure 6a. On the abscissa, we see the hydration level of the hydrogel. The water content ranges from completely dry (e.g. after freeze-drying) to completely wet (i.e. immersed in a water bath). In the envisioned application of a bike helmet cover, the hydration can range from the air moisture level to direct rain contact. In ordinate direction, the light intensity level is depicted, which ranges continuously from dark to direct solar irradiation. The hydrogel can swell and deswell due to the light intensity stimulus only if the gel is hydrated. Every continuous state transition is assumed to be reversible.

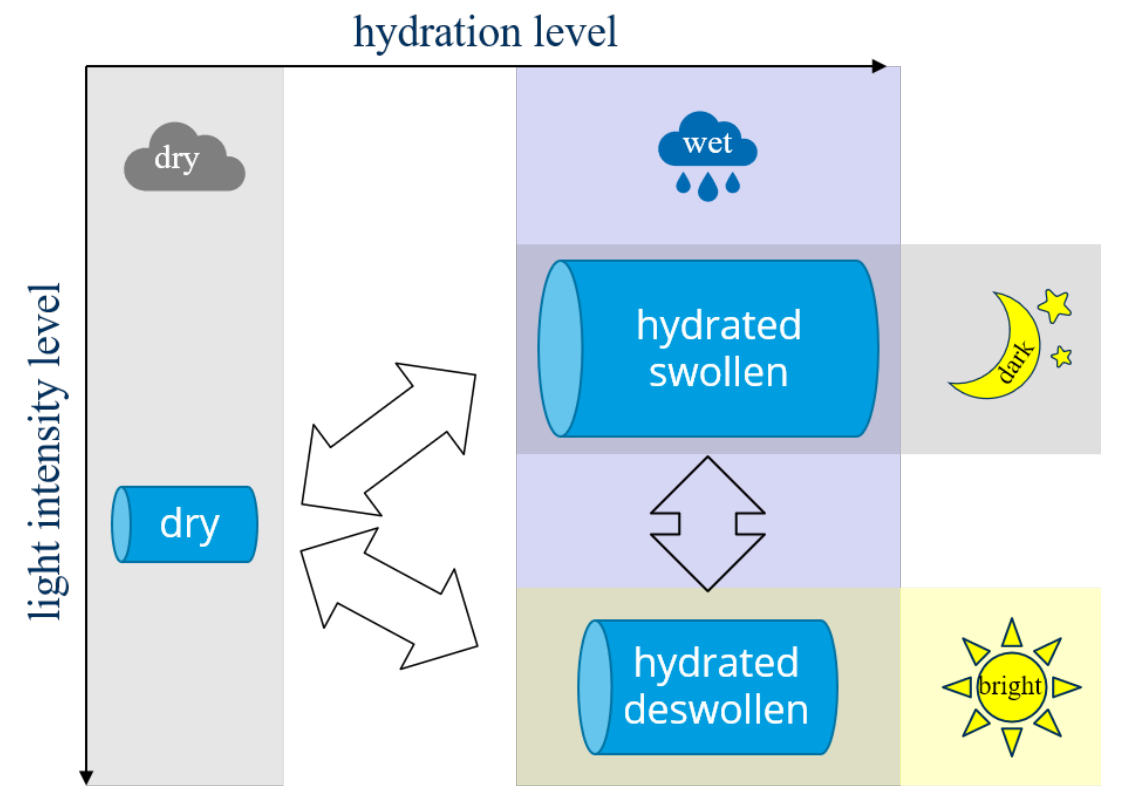

(a) The envisioned material provides three states of actuation for application.

\begin{tabular}{c|c|c} 
wet & light & swollen \\
\hline 0 & 0 & 0 \\
0 & 1 & 0 \\
1 & 0 & 1 \\
1 & 1 & $0(1 / 2)$
\end{tabular}

(b) Logic NIMPLY/inhibit gate

Figure 6: (a) Different states of the hydrogel for dry, deswollen and swollen state. When appropriate thresholds are defined, the hydrogel as an intrinsically logical material forms an inhibit gate behavior as depicted in (b).

Due to the mesh design (layer thicknesses and mesh width), we can now fix thresholds to digitize the continuous hydration and brightness. This leads to a logic table according to Figure 6b, i.e., an inhibit behavior. If the thresholds are adequately defined, the mesh is still open in partly swollen state (wet $\uparrow$ and brightness $\uparrow$ ). For our application, this translates to the following case that in hydrated state (after the rain) the mesh opens if the sun comes out again. If the mesh is dry, it is always opened.

\subsection{Modeling considerations}

In the current work, we use the Normalized Extended Temperature Expansion Model to reflect the swelling and deformation behavior of hydrogels inside structures. ${ }^{17}$

The Temperature Expansion Model is based on the analogy between hydrogel swelling and classical thermal expansion. Both phenomena lead to a stress-free isotropic expansion which is caused by an external stimulus. 
They can therefore both be described by using the same set of equations, i.e. the mechanical balance laws, kinematic relation and material behavior. Since the expansion due to hydrogel swelling can be very large, we use nonlinear geometry, i.e. the HENCKY strain $\varepsilon_{k l}^{\mathrm{H}}$ as a measure of deformation.

This leads to a set of partial differential equations

$$
\begin{array}{r}
\sigma_{k l, k}=0 ; \quad \sigma_{k l}=\sigma_{l k} \\
\varepsilon_{k l}^{\mathrm{H}}=\frac{1}{2} \ln B_{k l} \\
\sigma_{k l}=E_{k l m n}\left(\varepsilon_{m n}^{\mathrm{H}}-\beta\left(F^{\mathrm{S}}\right) \Delta F^{\mathrm{S}}\right) .
\end{array}
$$

Here, $\sigma_{k l}$ denotes the stress tensor, $B_{k l}$ is the left CAUCHY-Green deformation tensor, $E_{k l m n}$ is the elasticity tensor, $\beta\left(F^{\mathrm{S}}\right)$ is the stimulus expansion coefficient and $\Delta F^{\mathrm{S}}=F^{\mathrm{S}}-1$ the stimulus difference. The indices are $k, l, m, n \in[x, y, z]$ and $\delta_{k l}$ denotes the KRONECKER-delta. For further information about the modeling background, please refer to our previous works. ${ }^{17,27,28}$ For additional information about the combination of hydrogels and passive materials, please refer e.g. to the following works. ${ }^{7,13-15}$

\subsection{Simulation of active fibers and meshes}

In order to obtain a deeper insight into the behavior of the layered fibers, we performed simplified Finite Element simulations based on the Normalized Extended Temperature Expansion Model. For the simplified single fiber setup depicted in Figure 7, due to symmetry we use a quarter of the structure with respective symmetry conditions. The simulations were performed in the consistent $\mu$ MKS system, the MISES stress is therefore measured in MPa.

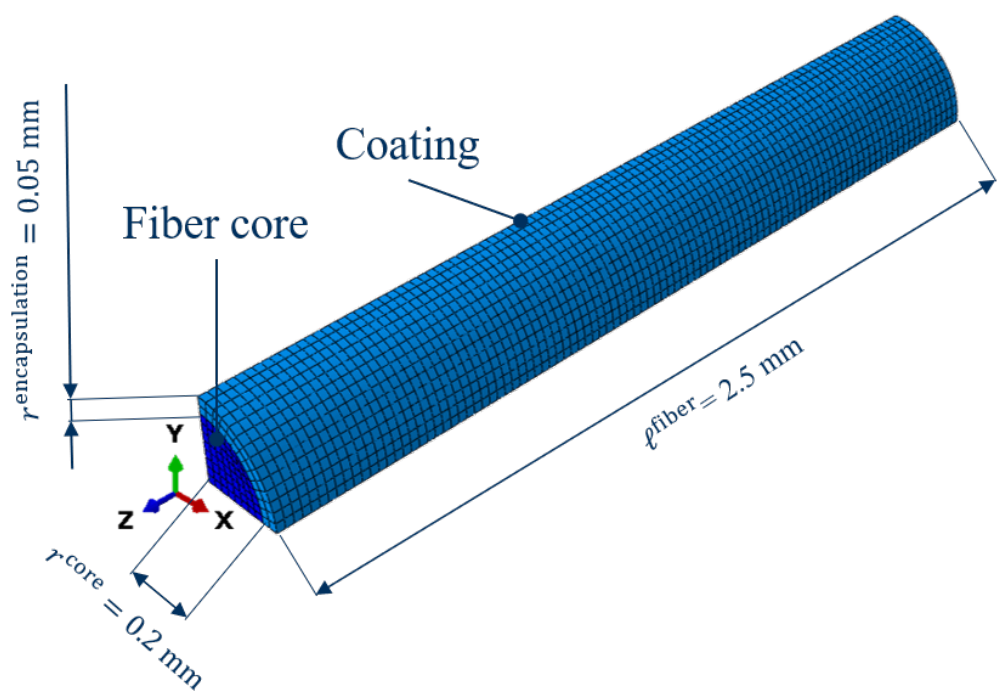

Figure 7: Simulation base model using symmetries.

Please note, that due to the prestretching of the mesh according to Figure 4, we do not expect buckling instability due to the swelling. However, future studies have to include these phenomena. In the current work, as described above, two different test cases with different composition of the core of the fiber, are investigated:

Case I In Figure $8 \mathrm{a}$, swelling simulation results for $\left(\beta \Delta F^{\text {Stimulus }}\right)=0.1$, i.e. $10 \%$ strain are depicted. The setup is chosen according to the single fiber representation with Case I (separated) as described in Figure 3a, i.e. the encapsulation has a thickness of $0.05 \mathrm{~mm}$. The fiber core was chosen with isotropic elastic modulus $E^{\text {fiber }} \gg E^{\text {hydrogel }}$. Due to the very thin layer, only very small thickness changes (here given in $x$-direction) result. 
Case II In Figure 8b, the model was changed to include the effect of the interpenetrated core. For the fiber, orthotropic material data according to KELLY ${ }^{40}$ was implemented, i.e., the material stiffness parameter in fiberlength direction is much higher than the ones in the other directions. In these preliminary simulation studies, the effect of the interpenetrating hydrogel was included by adding anisotropic expansion capabilities to the fiber. In further studies, the behavior of the combined material has to be represented using the composite theory. However, the basic approach already shows that the displacement of a point of the encapsulation is notably higher. This is due to the higher amount of material with swelling capabilities in radial direction.

\section{Case I with passive core}
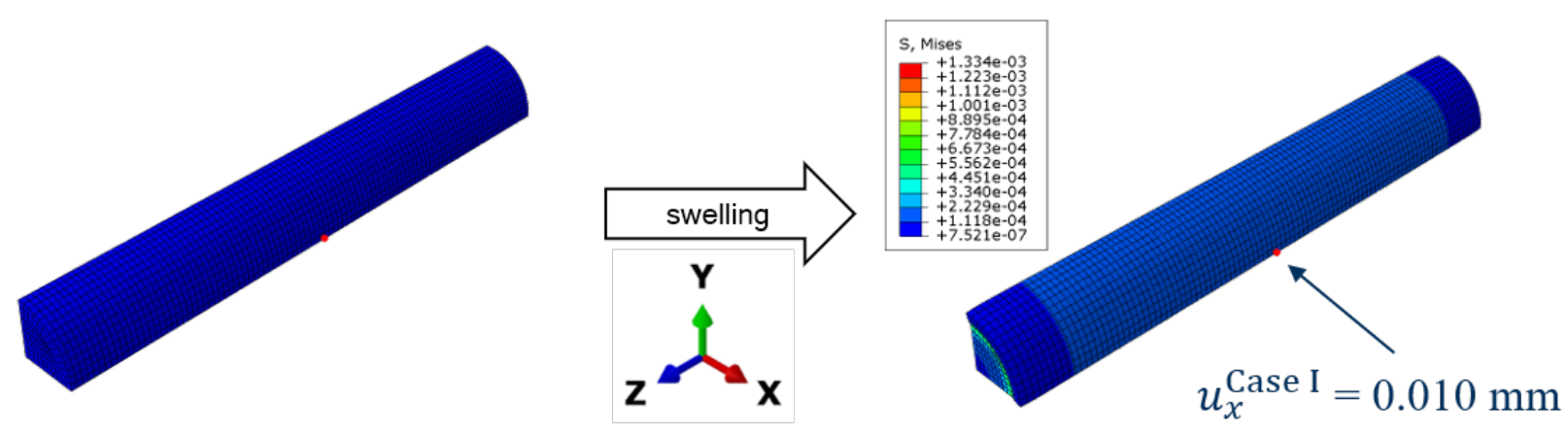

(a) Finite Element results for the swelling of a single fiber according to Case I (see Figure 3a) with a passive core.

\section{Case II with interpenetrated hydrogel/fiber in the core}


(b) Finite Element results for the swelling of a single fiber according to Case II (see Figure 3a). Here, the fiber core has both anisotropic elastic and swelling capacities.

Figure 8: Simulation results for (a) Case I and (b) Case II. In both cases, the simulations were performed in Abaqus according to the implementation of the Normalized Extended Temperature Expansion Model. In both models, 9100 quadratic hexahedral elements of type C3D20R were used. 


\section{CONCLUSION AND OUTLOOK}

In the current work, we have shown preliminary results for the design of hydrogel-coated meshes. The experimental base system is made of polyethylene fibers that are coated with a PAAm/PAMPS hydrogel with SIPN structure. The active mesh system is capable of performing swelling under hydration and can therefore serve as a starting point for the functionalization to allow light-sensitivity. The preliminary simulation results based on the Normalized Extended Temperature Expansion Model have highlighted the difference in composition of the active fibers: In Case I, coating and fiber are separated. This leads to a much smaller swelling and is not preferred for adhesion of the layers. In Case II, the hydrogel network forms both inside and outside the fiber. This results in a much better and more reliable adhesion. The Finite Element simulations in the current work have shown, that this also leads to much better swelling capacities for the fibers.

For the application in a self-closing rain cover, some important points remain to be discussed: Rain in industrial areas is mostly contaminated with chemicals and particles. This will likely impede the swelling capacities of the hydrogel. Also, the next step is the integration of the light responsiveness which can be achieved with another polymer or through copolymerization. At the modeling side, a composite model with passive fiber and active matrix can be derived to better represent the interpenetrating fiber and hydrogel networks in the active compound. Then, the complete mesh setup can be simulated in order to design the required functionalities.

Starting from the active hydrogel meshes as presented in the current work, the next step can be smart textiles that harness the power of hydrogels: The adaptation to combinations of stimuli - like humidity, temperature and brightness - that define environments.

\section{ACKNOWLEDGMENTS}

The present work is supported by the Deutsche Forschungsgemeinschaft (DFG) in the framework of the research training groups DFG-GRK 1865 "Hydrogel-based microsystems" and DFG-GRK 2430 "Interactive Fiber Rubber Composites" at Technische Universität Dresden.

\section{REFERENCES}

[1] Gerlach, G. and Arndt, K.-F., [Hydrogel sensors and actuators: engineering and technology], vol. 6, Springer Science \& Business Media (2009).

[2] Guelch, R. W., Holdenried, J., Weible, A., Wallmersperger, T., and Kroeplin, B., "Electrochemical stimulation and control of electroactive polymer gels", in [Smart Structures and Materials 2001: Electroactive Polymer Actuators and Devices], 4329, 328-334, International Society for Optics and Photonics (2001).

[3] Messing, R. and Schmidt, A. M., "Perspectives for the mechanical manipulation of hybrid hydrogels", Polymer Chemistry 2(1), 18-32 (2011).

[4] Bar-Cohen, Y., [Electroactive Polymers as Artificial Muscles: Capabilities, Potentials and Challenges], ch. 23, 188-196, American Society of Civil Engineers (2000).

[5] Kuo, J.-C., Huang, H.-W., Tung, S.-W., and Yang, Y.-J., "A hydrogel-based intravascular microgripper manipulated using magnetic fields", Sensors and Actuators A: Physical 211, 121-130 (2014).

[6] Binder, S. and Gerlach, G., "Intramolecular force-compensated hydrogel-based sensors with reduced response times", tm-Technisches Messen 86(4), 227-236 (2019).

[7] Ehrenhofer, A., Bingel, G., Paschew, G., Tietze, M., Schröder, R., Richter, A., and Wallmersperger, T., "Permeation control in hydrogel-layered patterned PET membranes with defined switchable pore geometry - experiments and numerical simulation", Sensors and Actuators B: Chemical 232, 499-505 (2016).

[8] Ehrenhofer, A. and Wallmersperger, T., "Active hydrogel composite membranes for the analysis of cell size distributions", in [Behavior and Mechanics of Multifunctional Materials XIII], Naguib, H. E., ed., 10968, 279 - 286, International Society for Optics and Photonics, SPIE (2019).

[9] Richter, A., Wenzel, J., and Kretschmer, K., "Mechanically adjustable chemostats based on stimuliresponsive polymers", Sensors and Actuators B: Chemical 125(2), 569 - 573 (2007).

[10] Ionov, L., "Hydrogel-based actuators: possibilities and limitations", Materials Today 17(10), 494-503 (2014). 
[11] Beebe, D. J., Moore, J. S., Bauer, J. M., Yu, Q., Liu, R. H., Devadoss, C., and Jo, B.-H., "Functional hydrogel structures for autonomous flow control inside microfluidic channels", Nature 404(6778), 588-590 (2000).

[12] Pan, H. M., Seuss, M., Neubauer, M. P., Trau, D. W., and Fery, A., "Tuning the mechanical properties of hydrogel core-shell particles by inwards interweaving self-assembly", ACS applied materials $\mathcal{E}$ interfaces $8(2)$, 1493-1500 (2016).

[13] Ehrenhofer, A., Hahn, M., Hofmann, M., and Wallmersperger, T., "Mechanical behavior and pore integration density optimization of switchable hydrogel composite membranes", Journal of Intelligent Material Systems and Structures 31(3), 425-435 (2020).

[14] Winkler, A., Ehrenhofer, A., Modler, N., Wallmersperger, T., and Gude, M., "Soft robotic structures by smart encapsulation of electronic devices", Submitted to Procedia Manufacturing (2020).

[15] Ehrenhofer, A. and Wallmersperger, T., "Shell-forming stimulus-active hydrogel composite membranes: Concept and modeling", Micromachines (2020).

[16] Ehrenhofer, A., Binder, S., Gerlach, G., and Wallmersperger, T., "Multisensitive swelling of hydrogels for sensor and actuator design", Advanced Engineering Materials (2020).

[17] Ehrenhofer, A., Elstner, M., and Wallmersperger, T., "Normalization of hydrogel swelling behavior for sensoric and actuatoric applications", Sensors and Actuators B: Chemical 255(Part 2), 1343 - 1353 (2018).

[18] Suzuki, A. and Tanaka, T., "Phase transition in polymer gels induced by visible light", Nature 346(6282), 345-347 (1990).

[19] Sumaru, K., Ohi, K., Takagi, T., Kanamori, T., and Shinbo, T., "Photoresponsive properties of poly (nisopropylacrylamide) hydrogel partly modified with spirobenzopyran", Langmuir 22(9), 4353-4356 (2006).

[20] Mamada, A., Tanaka, T., Kungwatchakun, D., and Irie, M., "Photoinduced phase transition of gels", Macromolecules 23(5), 1517-1519 (1990).

[21] Wang, Z. J., Li, C. Y., Zhao, X. Y., Wu, Z. L., and Zheng, Q., "Thermo-and photo-responsive composite hydrogels with programmed deformations", Journal of materials chemistry B 7(10), 1674-1678 (2019).

[22] Ashir, M., Hahn, L., Kluge, A., Nocke, A., and Cherif, C., "Development of innovative adaptive 3d fiber reinforced plastics based on shape memory alloys", Composites Science and Technology 126, 43-51 (2016).

[23] Haines, C. S., Lima, M. D., Li, N., Spinks, G. M., Foroughi, J., Madden, J. D. W., Kim, S. H., Fang, S., Jung de Andrade, M., Göktepe, F., Göktepe, Ö., Mirvakili, S. M., Naficy, S., Lepró, X., Oh, J., Kozlov, M. E., Kim, S. J., Xu, X., Swedlove, B. J., Wallace, G. G., and Baughman, R. H., "Artificial muscles from fishing line and sewing thread", Science (New York, N.Y.) 343(6173), 868-872 (2014).

[24] Pfeil, S., Katzer, K., Kanan, A., Mersch, J., Zimmermann, M., Kaliske, M., and Gerlach, G., "A biomimetic fish fin-like robot based on textile reinforced silicone", Micromachines 11(3) (2020).

[25] Wendler, J., Maraite, D., Nocke, A., and Cherif, C., "Novel textile moisture sensors based on multi-layered braiding constructions", Textile Research Journal 90(3-4), 469-477 (2020).

[26] Pollini, M., Paladini, F., Sannino, A., and Maffezzoli, A., "Development of hybrid cotton/hydrogel yarns with improved absorption properties for biomedical applications", Materials science $\&$ engineering. $C$, Materials for biological applications 63, 563-569 (2016).

[27] Ehrenhofer, A. and Wallmersperger, T., "Hydrogels for engineering: normalization of swelling due to arbitrary stimulus", in [Proc. SPIE 10163, Electroactive Polymer Actuators and Devices XIX], Proc. SPIE 10163, 1016321-15 (2017).

[28] Ehrenhofer, A. and Wallmersperger, T., "A normalization concept for smart material actuation by the example of hydrogels", PAMM 18(1), e201800176 (2018).

[29] Sobczyk, M. and Wallmersperger, T., "Modeling and simulation of the electro-chemical behavior of chemically stimulated polyelectrolyte hydrogel layer composites", Journal of Intelligent Material Systems and Structures 27(13), 1725 - 1737 (2016).

[30] Lucantonio, A., Nardinocchi, P., and Teresi, L., "Transient analysis of swelling-induced large deformations in polymer gels", Journal of the Mechanics and Physics of Solids 61(1), 205-218 (2013).

[31] Rossi, M., Nardinocchi, P., and Wallmersperger, T., "Swelling and shrinking in prestressed polymer gels: an incremental stress-diffusion analysis", Proceedings of the Royal Society A 475(2230), 20190174 (2019). 
[32] Bluhm, J., Serdas, S., and Schröder, J., "Theoretical framework of modeling of ionic EAPs within the theory of porous media", Archive of Applied Mechanics 86(1-2), 3-19 (2016).

[33] Leichsenring, P. and Wallmersperger, T., "Modelling and simulation of the chemically induced swelling behavior of anionic polyelectrolyte gels by applying the theory of porous media", Smart Materials and Structures 26(3), 035007 (2017).

[34] Ehlers, W., Acartürk, A., and Karajan, N., "Advances in modelling saturated soft biological tissues and chemically active gels", Archive of Applied Mechanics 80(5), 467-478 (2010).

[35] Drozdov, A., Sommer-Larsen, P., Christiansen, J. d., and Sanporean, C.-G., "Time-dependent response of hydrogels under constrained swelling", Journal of Applied Physics 115(23), 233517 (2014).

[36] Schneider, C. A., Rasband, W. S., and Eliceiri, K. W., "NIH image to Imagej: 25 years of image analysis", Nature methods 9(7), 671-675 (2012).

[37] Erfkamp, J., Guenther, M., and Gerlach, G., "Hydrogel-based piezoresistive sensor for the detection of ethanol", Journal of Sensors and Sensor Systems 7, 219-226 (04 2018).

[38] Binder, S., Krause, A. T., Voit, B., and Gerlach, G., "Bisensitive hydrogel with volume compensation properties for force compensation sensors", IEEE Sensors Letters 1(6), 1-4 (2017).

[39] Binder, S. and Gerlach, G., "Semi-interpenetrating polymer networks based on n-isopropylacrylamide and 2-acrylamido-2-methylpropane sulfonic acid for hydrogel-based chemical sensors", ECS Meeting Abstracts MA2020-01, 2426-2426 (05 2020).

[40] Kelly, A. and Zweben, C. H., [Comprehensive composite materials], Elsevier (2000). 